\title{
THE ENERGETIC AND EXPERIMENTAL BASED APPROACH TO DESCRIPTION OF BASIC MATERIAL CHARACTERISTICS AND MECHANICAL PROPERTIES OF SELECTED POLYMERS
}

\author{
Dariusz Kurpisz, Maciej Obst \\ Poznan University of Technology, Faculty of Mechanical Engineering, Poznan, Poland \\ e-mail: dariusz.kurpisz@put.poznan.pl; maciej.obst@put.poznan.pl
}

\begin{abstract}
The paper presents a proposal of using an experimental phenomenological approach and energy based method to the modelling of mechanical properties of nonlinear elastic materials using examples of two selected polymers. On the basis of an experimental stress-strain relation, together with transversal deformation measurement and the geometrical interpretation of the deformation process, analytical forms of the strain energy density function and a pure volumetric part of the strain energy density function have been introduced. The volumetric part of energy has been used in the description of the material damage process interpreted as the appearance of the first plastic deformations, which is the original part of the work and continuous investigations carried out by Wegner and Kurpisz. All theoretical investigations have been illustrated using examples of PVC and PA-6.
\end{abstract}

Keywords: nonlinear-elastic materials, energy based model, polymers, material damage

\section{Introduction}

Plastic materials are very widely used in the engineering practice due to some important construction properties like small weight, workability, and quite good mechanical properties. Modern complex mechanisms and structures as well as mechatronics devices are in the majority made of modern materials such as polymers. It is necessary and inevitable to do research on mechanical properties of polymers and to model plastic and other material behavior under diverse loads.

However, there exist a lot of physical models of plastic materials like (Necas and Hlavácek, 1981; Obst et al., 2015; Valavala, 2008; Ward and Sweeny, 2013). The identification of the reasons of material damage is generally very difficult. The tools or parts made of such materials are very often under influence of external forces, which generate complex stress states. The influence of dynamic loading compression at room temperature, when considering "Young's modulus, energy dissipation of plastic materials", was discussed in (Nakai and Yokoyama, 2008) for ABS, PA-6, PA-66, PC and in (Nakai and Yokoyama, 2015) relating to ABS, HDPE, PC, POM, PP and PVC. A variety of factors (Cowie and Arrighi, 1995) which should be taken into consideration, render the strain energy based mathematical modelling as one of the most important tools in the description of materials behaviour. Many researchers use the strain energy based methods all the time (Li, 2001; Wegner and Kurpisz, 2013; Wegner and Obst, 2007) but generally in the case of metals (Necas and Hlavácek, 1981; Wegner and Obst, 2007), metal foams (Wegner and Kurpisz, 2013), concrete ( $\mathrm{Li}$ and Ren, 2009), and also materials brittle as rocks (Hamiel et al., 2011). However, some also apply it in the modelling of plastic incompressible materials such as rubber (Sang et al., 2014).

It is very important to consider the possibility of taking an energetic approach when modelling materials in a nonlinear relation between stress and strain in the range of elastic deformations. This type of problems was investigated by Agostiniani and DeSimone (2012), based on 
the Ogden conception energy model which was used in the modelling of the nonlinear hyper-elastic material like rubber. However, this type of approach can be useful for other materials, for example, polymers and biological tissues.

The main aim of the current paper is the proposal of energy based and phenomenological approaches taking into consideration the description of selected mechanical experimental characteristics of two selected types of popular polymers: PVC and PA6, widely used in the engineering practice. Based on the stress-strain relation in a one-axial tension test with the unloading process and the relation between transversal and longitudinal deformations, the strain energy density function and its elastic part has been introduced. To ascertain the strain energy density function in a three-axial stress state, a geometrical interpretation of the deformation state has been used. The extraction of the purely volumetric part of strain energy was made on the basis of hydrostatic pressure interpretation, which is an original part of the paper.

For clarity, the work is divided into two parts. The first one focuses on the description of experimental results and the second one introduces basic analytical relations of the strain energy based polymer material model. All investigations are illustrated by simple examples.

\section{Messauring methods and experimental results}

The choice of experimental methods is strictly connected with the information which is possible to gain during basic strength tensile tests. In the aspects of mathematical model construction, the following issues are very important:

- determination of the stress strain relation both in the case of monotonic loading as in the case of unloading process,

- determination of the transversal-longitudinal deformation relation.

All experiments were conducted by making use of ZWICK Z100 strength tensile testing machine (monotonic loading) and hydraulic vibrator MTS (cyclic loading process). In all cases, elongation of the specimen was measured by an extensometr. The real loading velocities were applied as $37 \mathrm{~mm} / \mathrm{min}\left(0.0137 \mathrm{~s}^{-1}\right)$ and $38 \mathrm{~mm} / \mathrm{min}\left(0.0141 \mathrm{~s}^{-1}\right)$, respectively, for PVC and PA6. Such velocity of deformation does not follow the change of microstructure of investigated polymers. During the experimental tests, the polymer bar specimens were used as shown in Fig. 1. Transversal deformations were measured by the use of a high-resolution camera. During

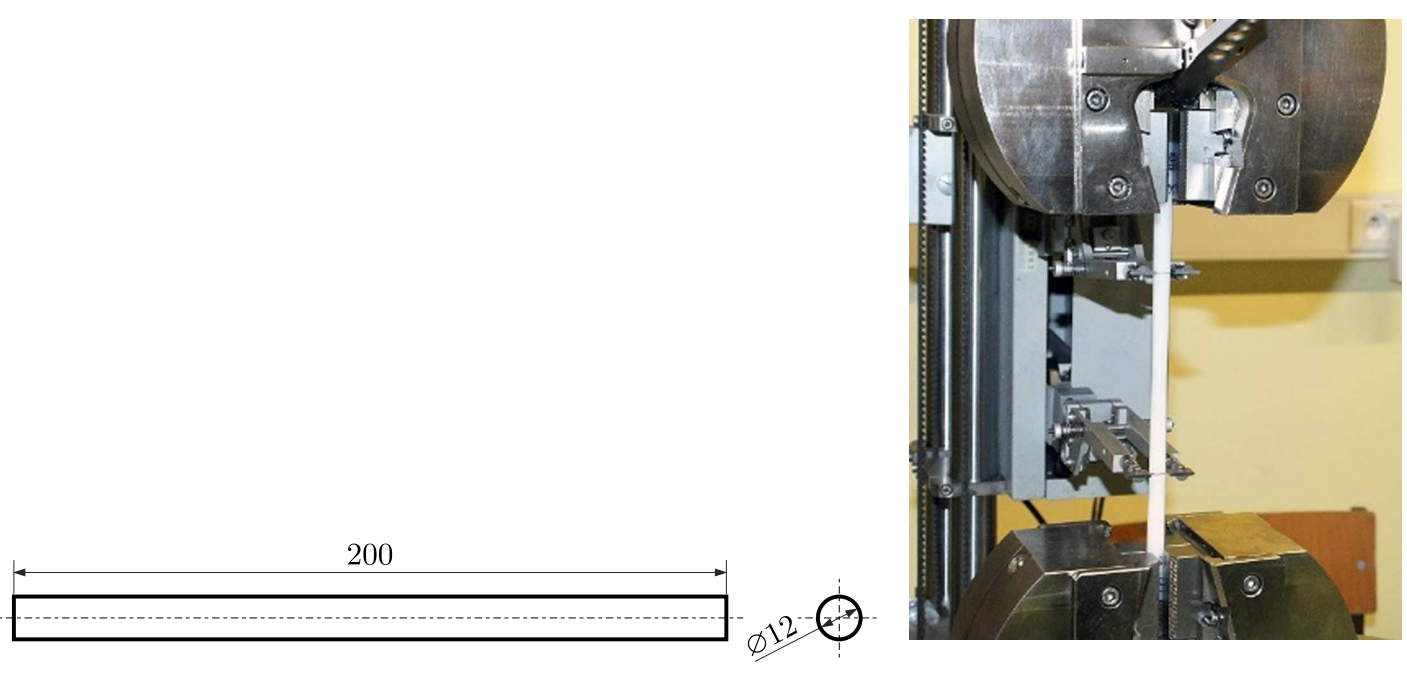

Fig. 1. Polymer specimen dimensions and the applied method of deformation measurement 
tension tests on the specimen surface, special reference marks were signified. On the basis of the measured longitudinal and transverse deformation, variable Poisson's ratio was estimated.

The basic experimental relations for the investigated polymers PVC and PA6 are presented below (Fig. 2).

(a)

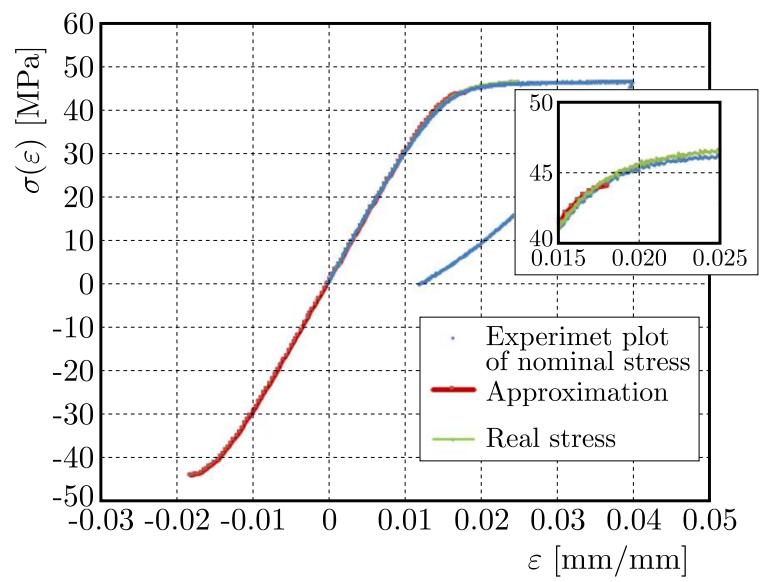

(b)

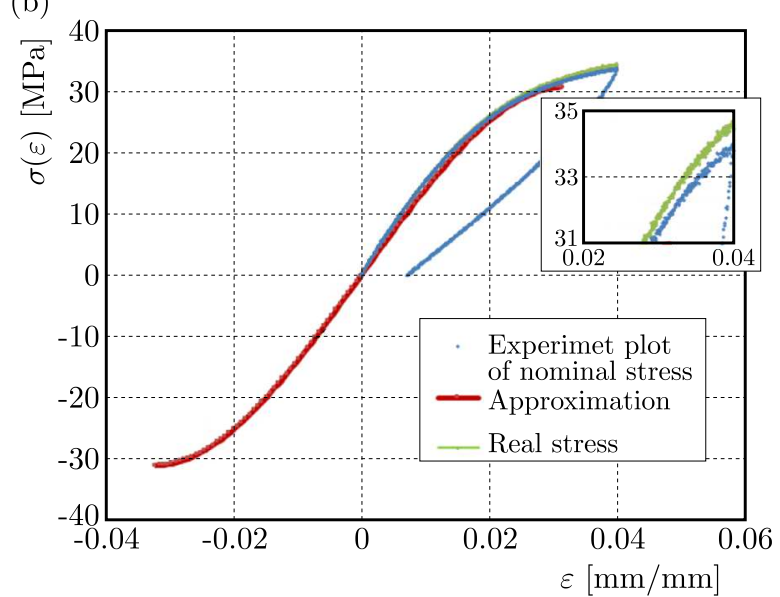

Fig. 2. Stress-strain relations in a monotonic one-axial tension process for polymers (a) PVC and (b) PA6

The shapes of the above experimental characteristics allow one to conclude that the discussed plastic materials are nonlinear elastic, and for small deformations, the nominal and real stress are approximately the same. In all cases, the upper yield limit of plastic flow does not exist.

Above, the experimental plots can be approximated by the use of the odd functions, respectively, for PVC and PA6

$$
\sigma\left(\varepsilon_{l}\right)= \begin{cases}3.06 \cdot 10^{3} \varepsilon_{l}-5.82 \cdot 10^{9} \varepsilon_{l}^{5} & \text { for } \quad \varepsilon_{l} \leqslant 0.018 \\ 1.45 \cdot 10^{3} \varepsilon_{l}-4.73 \cdot 10^{5} \varepsilon_{l}^{3} & \text { for } \quad \varepsilon_{l} \leqslant 0.032\end{cases}
$$

The changeable longitudinal deformation coefficient (tangential Young's modulus) for PVC and PA-6, can be written respectively as

$$
E\left(\varepsilon_{l}\right)=\frac{d \sigma}{d \varepsilon_{l}}=\left\{\begin{array}{l}
3.06 \cdot 10^{3}-29.1 \cdot 10^{9} \varepsilon_{l}^{4} \\
1.45 \cdot 10^{3}-14.9 \cdot 10^{5} \varepsilon_{l}^{2}
\end{array}\right.
$$

Because relations (2.2) are true only in the case of elastic deformations, the next step was to experimentally determine the range of elastic deformations.

Because the elastic deformations occur when the residual deformations, after the unloading process, equal zero (in practice does not exceed 0.002), it is necessary to realize this process for discussed materials. The experiment was made by the use of a hydraulic pulse generator MTS. Each of two investigated specimens were applied to a single loading and unloading process, where the current values of stress and deformations were measured in real time. A few initiation levels were applied to PVC and PA6 levels for the unloading: 0.04, 0.035, 0.03, 0.025, 0.02, 0.015 and 0.01 (Fig. 3). In order to find the elastic deformation range, we needed to choose the loading process where the residual deformations did not exceed zero.

It was assumed that the deformation was included in the elastic range if its residual value did not exceed 0.002 , therefore, the initial deformation (at the beginning of the unloading process) did not exceed the level P4 $(\varepsilon<0.02)$ for PVC and P3 $(\varepsilon<0.015)$ for PA6.

The experimental plots of the relation between transversal $\varepsilon_{d}$ and longitudinal $\varepsilon_{l}$ deformations are given below (Fig. 4). 
(a)

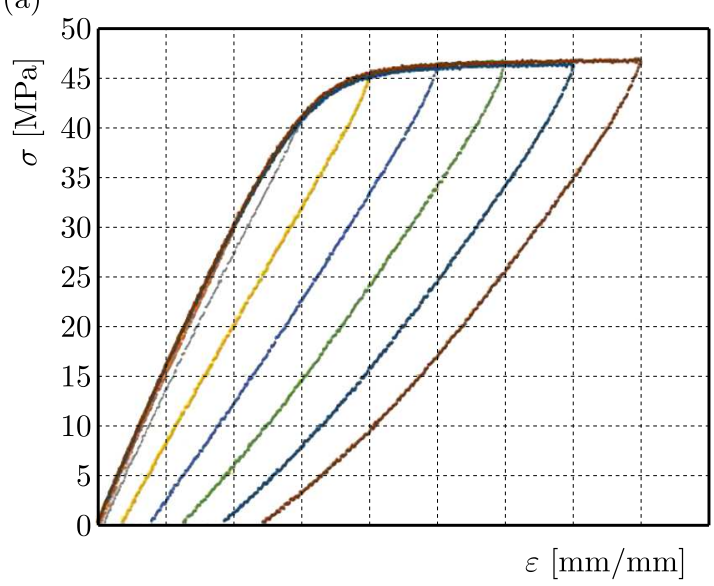

(b)

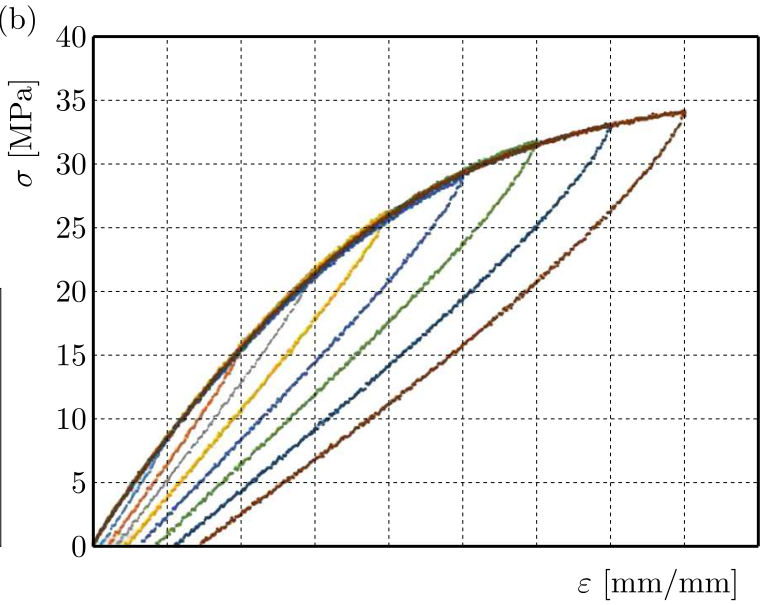

Fig. 3. The stress-strain relations in the one-axial tension process with cyclic unloading for (a) PVC and (b) PA6

(a)

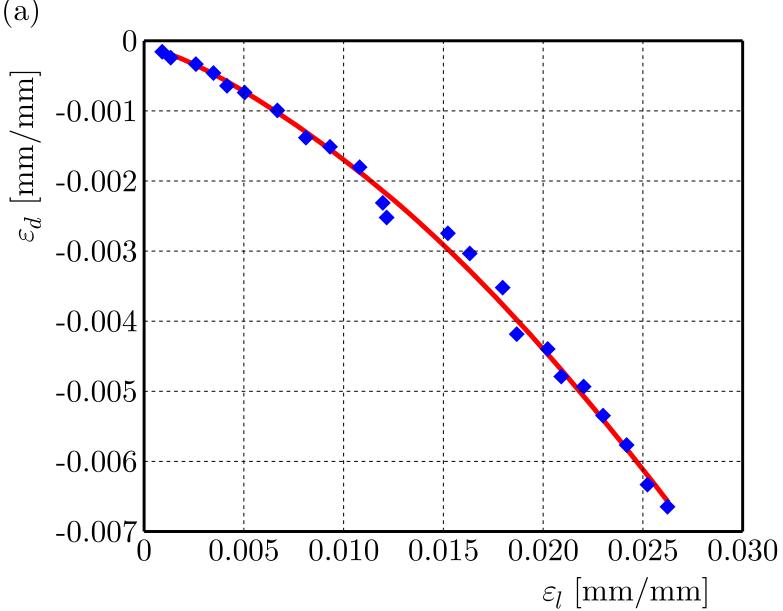

(b)

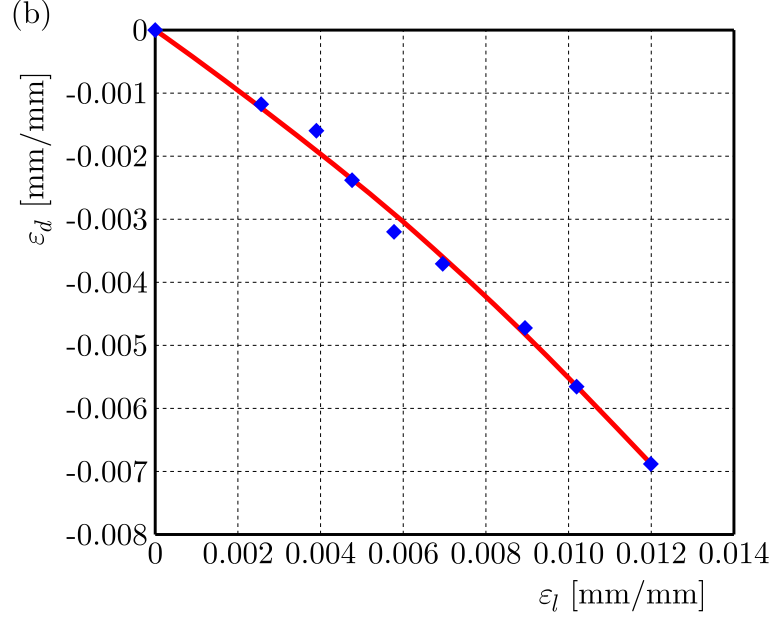

Fig. 4. Transversal deformations coefficients (blue points) and the approximations (red lines) for (a) PVC and (b) PA6

The approximations of the experimental results can be expressed as

$$
\varepsilon_{d}\left(\varepsilon_{l}\right)=-5.1 \varepsilon_{l}^{2}-0.116 \varepsilon_{l} \quad v\left(\varepsilon_{l}\right)=-\frac{d \varepsilon_{d}}{d \varepsilon_{l}}=10.2 \varepsilon_{l}+0.116
$$

for PVC, and

$$
\varepsilon_{d}\left(\varepsilon_{l}\right)=-0.49 \varepsilon_{l} \quad v\left(\varepsilon_{l}\right)=-\frac{d \varepsilon_{d}}{d \varepsilon_{l}}=0.49
$$

for PA6.

\section{Strain energy based description of polymers - experimental results}

As mechanical properties of the material are independent of the sample geometry, it is very important to use this type of characteristic which has no relation to geometry. Such type of measure, especially in one-axial tension tests, is density of deformation work, interpreted as the work of external loads under an elementary volume of a piece of the material. 
Basing on the experimental stress-strain relation, we can write $\left(W(\varepsilon)\right.$ in $\left.\mathrm{MJ} / \mathrm{m}^{3}\right)$

$$
W(\varepsilon)=\frac{1}{V_{0}} \int_{0}^{\Delta L} F(l) d l=\int_{0}^{\Delta L} \frac{F(l)}{A_{0}} d\left(\frac{l}{L_{0}}\right)=\int_{0}^{\varepsilon} \sigma(\varepsilon) d \varepsilon
$$

where: $L_{0}$ - initial length of the specimen, $A_{0}$ - initial cross-section of the specimen, $V_{0}$ - initial volume of the specimen.

For each unload initiation level, the elastic part of energy was calculated. It was assumed that the value of energy was calculated as the area under the unloading curve. Hence, the pattern was used

$$
W^{e}\left(\varepsilon_{l}\right)=\int_{0}^{\varepsilon_{l}} \sigma_{u}(\varepsilon) d \varepsilon
$$

where: $W^{e}\left(\varepsilon_{l}\right)\left[\mathrm{MJ} / \mathrm{m}^{3}\right]$ is the whole elastic part of energy for unloading level $1, \sigma_{u}(\varepsilon)[\mathrm{MPa}]-$ stress strain relation during unloading, $\varepsilon_{l}[\mathrm{~mm} / \mathrm{mm}]$ - level of the unloading process initiation.

The plots of elastic parts of the strain energy density functions for the discussed polymers are illustrated in Fig. 5.
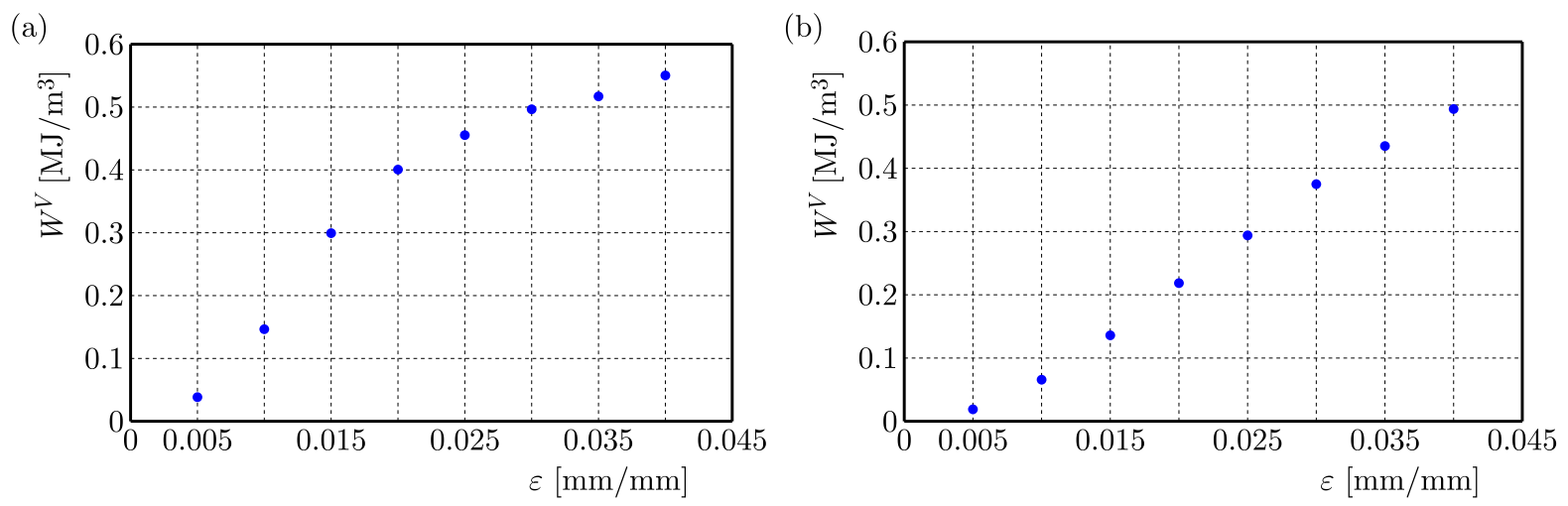

Fig. 5. Plots of elastic parts of strain energy for polymers (a) PVC and (b) PA6

\section{Geometrical interpretation of the deformation process - strain energy density function in a complex load state of polymer}

Every deformation process can be illustrated by the use of a deformation path $C$ which is a set of points (located in the three-axial space of principal deformation state components) represented by subsequent deformation states. The change of the deformation state is also motion along the path $C$. During the motion, the principal stress components are changeable.

To introduce an analytical form of the strain energy density function based on a phenomenological approach, let us look at the following assumptions:

- The deformation process occurs slowly, so the kinetic effects can be neglected

- The temperature is constant and does not change

- The external forces are in range of elastic deformations

- The material is nonlinear elastic, so the longitudinal and transversal deformation coefficients are functions of the current strain. 
The deformation path can be expressed as

$$
C=\left\{\begin{array}{l}
\varepsilon_{1}=\varphi_{1}(t) \\
\varepsilon_{2}=\varphi_{2}(t) \\
\varepsilon_{3}=\varphi_{3}(t)
\end{array} \quad \text { for } \quad t \in\langle 0,1\rangle\right.
$$

Motion along the path $C$ is a consequence of the influence of external loads or equivalent the change of principal stress components from the beginning (virgin) point to the final deformation state (Fig. 6).

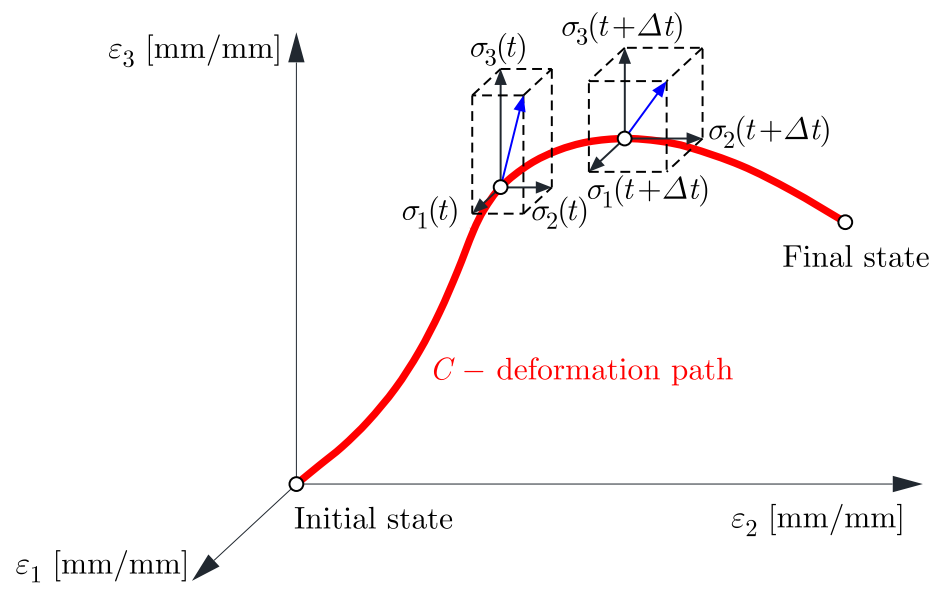

Fig. 6. Deformation process in the space of principal deformation state components

In the range of elastic deformations, the relations between stress and strain take the differential form

$$
\begin{aligned}
& \varepsilon_{1}^{\prime}=\frac{\sigma_{1}^{\prime}(t)}{E\left(\varepsilon_{1}\right)}-v\left(\varepsilon_{2}\right) \frac{\sigma_{2}^{\prime}(t)}{E\left(\varepsilon_{2}\right)}-v\left(\varepsilon_{3}\right) \frac{\sigma_{3}^{\prime}(t)}{E\left(\varepsilon_{3}\right)} \\
& \varepsilon_{2}^{\prime}=\frac{\sigma_{2}^{\prime}(t)}{E\left(\varepsilon_{2}\right)}-v\left(\varepsilon_{1}\right) \frac{\sigma_{1}^{\prime}(t)}{E\left(\varepsilon_{1}\right)}-v\left(\varepsilon_{3}\right) \frac{\sigma_{3}^{\prime}(t)}{E\left(\varepsilon_{3}\right)} \\
& \varepsilon_{3}^{\prime}=\frac{\sigma_{3}^{\prime}(t)}{E\left(\varepsilon_{3}\right)}-v\left(\varepsilon_{2}\right) \frac{\sigma_{2}^{\prime}(t)}{E\left(\varepsilon_{2}\right)}-v\left(\varepsilon_{1}\right) \frac{\sigma_{1}^{\prime}(t)}{E\left(\varepsilon_{1}\right)}
\end{aligned}
$$

Because the range of elastics deformations is very small, so the principal stress and strain directions can be assumed to be the same.

On the base of (4.2), we can write that

$$
\begin{aligned}
\sigma_{i}^{\prime(t)} & =E\left(\varepsilon_{i}\right) \frac{\varepsilon_{i}^{\prime} \prod_{k=1}^{3}\left[1+v\left(\varepsilon_{k}\right)\right]+\left[1+v\left(\varepsilon_{i}\right)\right] \prod_{k=1}^{3} v\left(\varepsilon_{k}\right)\left(\varepsilon_{k}^{\prime}-\varepsilon_{i}^{\prime}\right)}{\prod_{k=1}^{3}\left[1+v\left(\varepsilon_{k}\right)\right]-\left[1+v\left(\varepsilon_{i}\right)\right]^{2}\left[\prod_{k=1}^{3} v\left(\varepsilon_{k}\right)-v\left(\varepsilon_{i}\right)+2 \frac{\prod_{k=1}^{3} v\left(\varepsilon_{k}\right)}{v\left(\varepsilon_{i}\right)}\right]} \\
+ & E\left(\varepsilon_{i}\right) \frac{\frac{1+v\left(\varepsilon_{i}\right)}{v\left(\varepsilon_{i}\right)} \prod_{k=1}^{3} v\left(\varepsilon_{k}\right)\left(\prod_{k=1}^{3} \varepsilon_{k}^{\prime}-3 \varepsilon_{i}^{\prime}\right)}{\prod_{k=1}^{3}\left[1+v\left(\varepsilon_{k}\right)\right]-\left[1+v\left(\varepsilon_{i}\right)\right]^{2}\left[\prod_{k=1}^{3} v\left(\varepsilon_{k}\right)-v\left(\varepsilon_{i}\right)+2 \frac{\prod_{k=1}^{3} v\left(\varepsilon_{k}\right)}{v\left(\varepsilon_{i}\right)}\right]}
\end{aligned}
$$

hence

$$
\begin{aligned}
\sigma_{i}(t) & =\int E\left(\varepsilon_{i}\right) \frac{\varepsilon_{i}^{\prime} \prod_{k=1}^{3}\left[1+v\left(\varepsilon_{k}\right)\right]+\left[1+v\left(\varepsilon_{i}\right)\right] \prod_{k=1}^{3} v\left(\varepsilon_{k}\right)\left(\varepsilon_{k}^{\prime}-\varepsilon_{i}^{\prime}\right)}{\prod_{k=1}^{3}\left[1+v\left(\varepsilon_{k}\right)\right]-\left[1+v\left(\varepsilon_{i}\right)\right]^{2}\left[\prod_{k=1}^{3} v\left(\varepsilon_{k}\right)-v\left(\varepsilon_{i}\right)+2 \frac{\prod_{k=1}^{3} v\left(\varepsilon_{k}\right)}{v\left(\varepsilon_{i}\right)}\right]} d t \\
& +\int E\left(\varepsilon_{i}\right) \frac{\frac{1+v\left(\varepsilon_{i}\right)}{v\left(\varepsilon_{i}\right)} \prod_{k=1}^{3} v\left(\varepsilon_{k}\right)\left(\prod_{k=1}^{3} \varepsilon_{k}^{\prime}-3 \varepsilon_{i}^{\prime}\right)}{\prod_{k=1}^{3}\left[1+v\left(\varepsilon_{k}\right)\right]-\left[1+v\left(\varepsilon_{i}\right)\right]^{2}\left[\prod_{k=1}^{3} v\left(\varepsilon_{k}\right)-v\left(\varepsilon_{i}\right)+2 \frac{\prod_{k=1}^{3} v\left(\varepsilon_{k}\right)}{v\left(\varepsilon_{i}\right)}\right]} d t
\end{aligned}
$$

where $\varepsilon_{i}=\varphi_{i}(t)$ for $i=1,2,3$. 
The density of the deformation work without loss of the generality can be written as a function of the principal deformation state components. Each deformation state can be replaced by an equivalent principal deformation state. Therefore, the deformation work can be written as

$$
W\left(\varepsilon_{1}, \varepsilon_{2}, \varepsilon_{3}\right)=\int \sum_{i=1}^{3} \sigma_{i} d \varepsilon_{i}=\int_{0}^{1} \sum_{i=1}^{3} \sigma_{i}(t) \varepsilon_{i}^{\prime}(t) d t
$$

for $i=1,2,3$.

Assuming that the deformation process goes along a straight line, equating

$$
\varphi_{i}(t)=\varepsilon_{i}^{F} t
$$

where $\varepsilon_{i}^{F}$ is the value of the final deformation state component for $i=1,2,3$, we can write that

$$
W\left(\varepsilon_{1}^{F}, \varepsilon_{2}^{F}, \varepsilon_{3}^{F}\right)=\int_{0}^{1} \sum_{i=1}^{3} \sigma_{i}\left(\varphi_{1}(t), \varphi_{2}(t), \varphi_{3}(t)\right) \varepsilon_{i}^{F} d t
$$

To extract the volumetric part of the strain energy density function, it can be assumed that the elementary volume piece of the material is under influence of hydrostatic pressure, and so

$$
\sigma_{i}\left(\varepsilon_{i}\right)=k s
$$

for $i=1,2,3$ and $s \in\langle 0,1\rangle$ is a non-dimensional coefficient which can be interpreted as the ratio of the current hydrostatic pressure to its basic value $k$ [MPa].

In such situations, the system of equations (3.2) can be simplified to the form

$$
\begin{aligned}
& \varepsilon_{1}^{\prime}=\frac{k}{E\left(\varepsilon_{1}\right)}-v\left(\varepsilon_{2}\right) \frac{k}{E\left(\varepsilon_{2}\right)}-v\left(\varepsilon_{3}\right) \frac{k}{E\left(\varepsilon_{3}\right)} \\
& \varepsilon_{2}^{\prime}=\frac{k}{E\left(\varepsilon_{2}\right)}-v\left(\varepsilon_{1}\right) \frac{k}{E\left(\varepsilon_{1}\right)}-v\left(\varepsilon_{3}\right) \frac{k}{E\left(\varepsilon_{3}\right)} \\
& \varepsilon_{3}^{\prime}=\frac{k}{E\left(\varepsilon_{3}\right)}-v\left(\varepsilon_{2}\right) \frac{k}{E\left(\varepsilon_{2}\right)}-v\left(\varepsilon_{1}\right) \frac{k}{\left.E\left(\varepsilon_{1}\right)\right)}
\end{aligned}
$$

According to isotropy of the discussed materials, the above system of equations can be expressed in the equivalent form

$$
\varepsilon^{\prime}=[1-2 v(\varepsilon)] \frac{k}{E(\varepsilon)}
$$

where $\varepsilon_{1}^{\prime}=\varepsilon_{2}^{\prime}=\varepsilon_{3}^{\prime}=\varepsilon^{\prime}(s)$.

Consequently, the deformation path takes the form

$$
C^{V}: \quad \varepsilon_{1}=\varepsilon_{2}=\varepsilon_{3}=\varepsilon=\alpha(s)
$$

Using relationship (3.2), it is possible to determine the shape of deformation path $C$ according to the principal stress coefficients

$$
C=\left\{\begin{array}{l}
\varepsilon_{1}=\beta_{1}(t) \\
\varepsilon_{2}=\beta_{2}(t) \\
\varepsilon_{3}=\beta_{3}(t)
\end{array}\right.
$$


The volume change in terms of (4.11) and (4.12) can be written, respectively, as

$$
\frac{\Delta V}{V}=[1+\alpha(s)]^{3}-1
$$

and

$$
\frac{\Delta V}{V}=\prod_{i=1}^{3}\left[1+\beta_{i}(t)\right]-1
$$

Comparing the right-hand sides of the above equations, we have

$$
[1+\alpha(s)]^{3}-1=\prod_{i=1}^{3}\left[1+\beta_{i}(t)\right]-1
$$

and after transformations

$$
s=\alpha^{-1}\left(\sqrt[3]{\prod_{i=1}^{3}\left[1+\beta_{i}(t)\right]}-1\right)=\gamma(t)
$$

Finally, the equation of the deformation path takes the form

$$
C^{V}: \quad \varepsilon_{1}=\varepsilon_{2}=\varepsilon_{3}=\varepsilon=\alpha(\gamma(t))=\sqrt[3]{\prod_{i=1}^{3}\left[1+\beta_{i}(t)\right]}-1
$$

The volumetric part of energy

$$
W^{V}\left(\varepsilon_{1}^{F}, \varepsilon_{2}^{F}, \varepsilon_{3}^{F}\right)=\int_{0}^{1} \sum_{i=1}^{3} \sigma_{i}(t) \alpha^{\prime}(\gamma(t)) \gamma^{\prime}(t) d t
$$

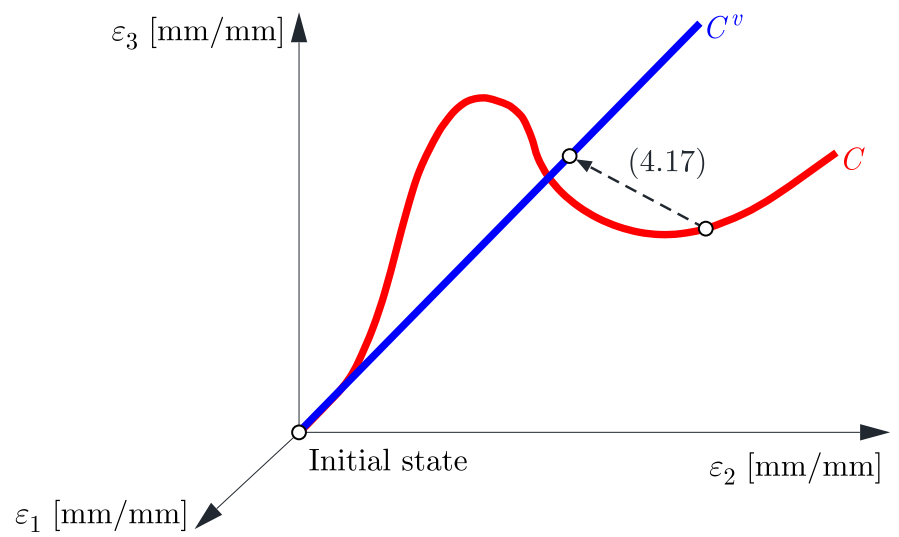

Fig. 7. The relation between the deformation path $C$ and its volumetric part $C^{V}$

\section{Example}

To illustrate the theoretical investigations, let us consider the experimental characteristics of PVC and PA6 polymers, which take the analytical form $(2.2)_{1},(2.3)_{2}$ and $(2.2)_{2},(2.4)_{2}$, respectively. 
Then, differential equation (4.10) takes the form

$$
\frac{d \varepsilon}{d s}=\left[1-2\left(a_{2} \varepsilon+b_{2}\right)\right] \frac{k}{a_{1}+b_{1} \varepsilon^{2 n-2}}
$$

where for PVC

$$
a_{1}=3.06 \cdot 10^{3} \quad b_{1}=-29.1 \cdot 10^{9} \quad a_{2}=10.2 \quad b_{2}=0.116 \quad n=3
$$

and for PA6

$$
a_{1}=1.45 \cdot 10^{3} \quad b_{1}=-14.9 \cdot 10^{5} \quad a_{2}=0 \quad b_{2}=0.49 \quad n=2
$$

After a series of transformations, the solution can be written as:

- for PVC

$$
\begin{gathered}
-\frac{b_{1}}{8 a_{2}} \varepsilon^{4}-\frac{b_{1}\left(1-2 b_{2}\right)}{12 a_{2}^{2}} \varepsilon^{3}-\frac{b_{1}\left(1-2 b_{2}\right)^{2}}{16 a_{2}^{3}} \varepsilon^{2}-\frac{b_{1}\left(1-2 b_{2}\right)^{3}}{16 a_{2}^{4}} \varepsilon \\
-\frac{1}{2 a_{2}}\left[a_{1}+\frac{b_{1}\left(1-2 b_{2}\right)^{2}}{16 a_{2}^{4}}\right] \ln \left(1-\frac{2 a_{2}}{1-2 b_{2}} \varepsilon\right)=k s
\end{gathered}
$$

- for PA6

$$
-\frac{b_{1}}{4 a_{2}} \varepsilon^{2}-\frac{b_{1}\left(1-2 b_{2}\right)}{4 a_{2}^{2}} \varepsilon-\frac{1}{2 a_{2}}\left[a_{1}+\frac{b_{1}\left(1-2 b_{2}\right)^{2}}{4 a_{2}^{2}}\right] \ln \left(1-\frac{2 a_{2}}{1-2 b_{2}} \varepsilon\right)=k s
$$

Taking into consideration the axial tension in direction 1 , we can write

$$
C:\left\{\begin{array}{l}
\varepsilon_{1}=\varepsilon_{1}^{F} t \\
\varepsilon_{2}=v(t) \varepsilon_{1}^{F} t \\
\varepsilon_{3}=v(t) \varepsilon_{1}^{F} t
\end{array}\right.
$$

and after using relations (4.17) and (4.18)

$$
W^{V}\left(\varepsilon_{1}^{F}\right)=\int_{0}^{1} \sigma_{1}(t) \alpha^{\prime}(\gamma(t)) \gamma^{\prime}(t) d t
$$

where

$$
\sigma_{1}(t)= \begin{cases}a_{1} \varepsilon_{1}^{F} t+\frac{1}{5} b_{1}\left(\varepsilon_{1}^{F} t\right)^{5} & \text { for PVC } \\ a_{1} \varepsilon_{1}^{F} t+\frac{1}{3} b_{1}\left(\varepsilon_{1}^{F} t\right)^{3} & \text { for PA-6 }\end{cases}
$$

and

$$
\alpha(\gamma(t))=\sqrt[3]{\prod_{i=1}^{3}\left[1+\beta_{i}(t)\right]}-1
$$

both for PVC and PA6.

After using the theoretical method and numerical calculations, we can indicate the results below (Fig. 8).

The plastic flow occurs, when the pure volumetric part of energy is decreasing and the value of volumetric energy is strictly connected with the transversal deformation coefficient.

The approximation in the range of initial (small) deformation can be expressed in the following form

$$
W^{V}\left(\varepsilon_{1}^{F}\right)= \begin{cases}661\left(\varepsilon_{1}^{F}\right)^{2}+0.80 \varepsilon_{1}^{F} & \text { for PVC } \\ 9.67\left(\varepsilon_{1}^{F}\right)^{2}+0.004 \varepsilon_{1}^{F} & \text { for PA-6 }\end{cases}
$$



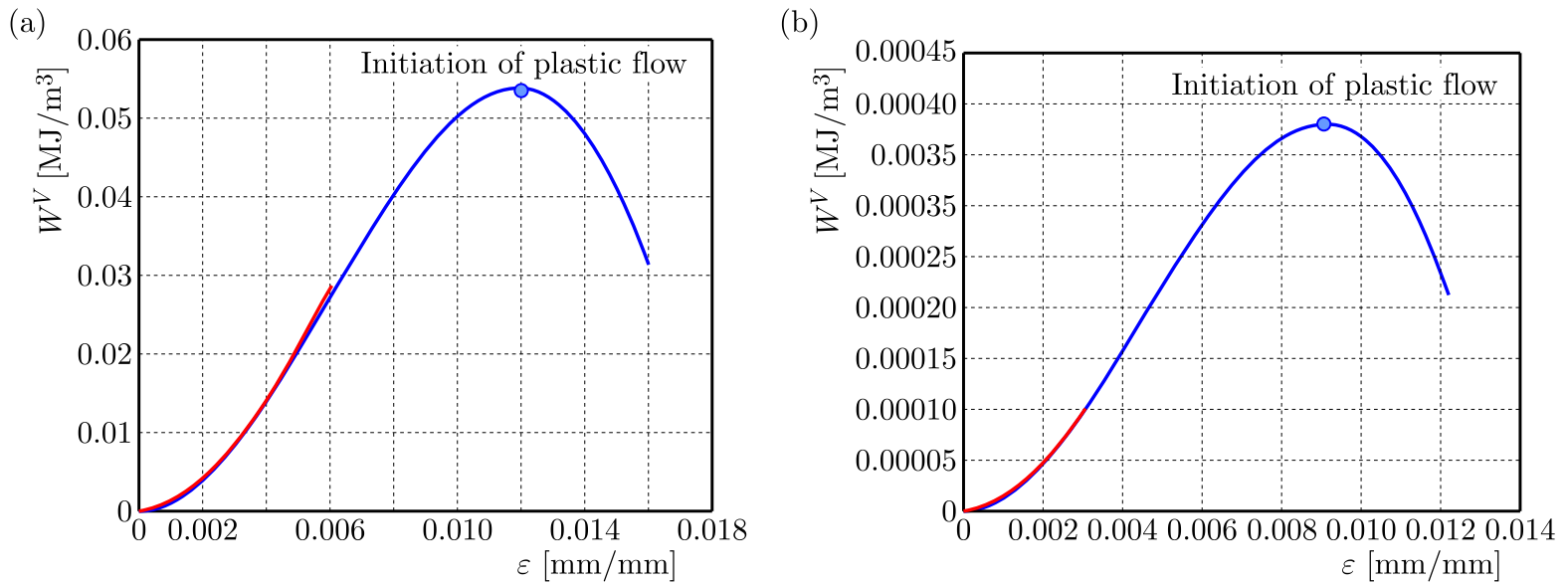

Fig. 8. Plots of pure volumetric parts of energy density (blue line) and its approximation (red line) for (a) PVC and (b) PA-6

\section{Conclusions}

Based on stress-strain relations and commonly carried out tensile tests completed by the unloading process and transverse deformation, the paper describes the strain energy density function whose elastic part of strain energy is assigned. The strain energy density and the appropriate elastic part of strain energy have been used as a phenomenological approach to describe the strain energy based deformation paths for PVC and PA6 polymers. Presented experimental results and analytical solutions allow us to conclude that the general meaning during deformation processes of the investigated polymers has the shear strain energy. The volumetric energy is a very small part of the strain energy, which means that the material deformation process is strictly connected with the change of the material shape. The results obtained show that the use of the strain energy density is a good tool in the description of material mechanical properties of polymers. On the grounds of procuring the experimental stress-strain relation and the transversal deformation, one can estimate and determine volumetric deformation. An important conclusion of the presented work is the fact that the initiation of plastic flow occurs when the pure volumetric part of energy begins to decrease (Fig. 8). Presented results prepared for a one-axis deformation state are interesting and valuable, but the multiaxial deformation state is more important in the engineering practice. In the future, the authors need to plan and perform more investigations focused on multiaxial deformation states for non-classic materials such as various polymers in different loading conditions. As it has been demonstrated, by using particular parts of the strain energy density function it is possible to solve many strength problems of non-classical materials. Presented in the paper the concept of the strain energy density based on investigation of the mechanical material state can be a promising idea for theoretical descriptions of properties of modern non-classic materials.

\section{Acknowledgements}

The presented research results, were funded with grants for education allocated by the Ministry of Science and Higher Education in Poland.

\section{References}

1. Agostiniani V., DeSimone A., 2012, Ogden-type energies for nematic elastomers, International Journal of Non-Linear Mechanics, 47, 402-412 
2. Cowie J.M.G., Arrighi V., 1995, Polymers: Chemistry and Physics of Modern Materials, CRC Press, Taylor \& Francis Group, Boca Raton

3. Hamiel Y., Lyakhovsky V., Ben-Zion Y., 2011, The elastic strain energy of damaged solids with applications to non-linear deformation of crystalline rocks, Pure and Applied Geophysics, 168, 2199-2210

4. Li J., REN X., 2009, Stochastic damage model for concrete based on energy equivalent strain, International Journal of Solids and Structures, 46, 2407-2419

5. Li Q.M., 2001, Strain energy density failure criterion, International Journal of Solids and Structures, 38, 6997-7013

6. Nakai K., Yokoyama T., 2008, Strain rate dependence of compressive stress-strain loops of several polymers, Journal of Solid Mechanics and Materials Engineering, 2, 4, 557-566

7. Nakai K., Yokoyama T., 2015, Uniaxial compressive response and constitutive modeling of selected polymers over a wide range of strain rates, Journal Dynamic Behavior Materials, 1, 15-27

8. Nechs J., Hlavácek I., 1981, Mathematical Theory of Elastic and Elasto Plastic Bodies: An Introduction, Elsevier

9. Obst M., Kurpisz D., Mencel K., 2015, Energy based mechanical characteristics of polymers POM-C, PET, PA6, PVC, PVDF, Machine Dynamics Research, 39, 4, 93-106

10. Sang J.B., Sun L.F., Xing S.F., Liu B.H., Sun Y.L., 2014, Mechanical properties of polymer rubber materials based on a new constitutive model, Polymers and Polymer Composites, 22, 8

11. Valavala P.K., 2008, Multiscale constitutive modeling of polymer materials, Dissertation, Michigan Technological University

12. Ward I.M., Sweeny J., 2013, Mechanical Properties of Solid Polymers, John Wiley \& Sons, Ltd., 3rd ed., ISBN: 9781444319507

13. Wegner T., Kurpisz D., 2013, Phenomenological modeling of mechanical properties of metal foam, Journal of Theoretical and Applied Mechanics, 51, 203-214

14. Wegner T., Obst M., 2007, The process of uniaxial stretching in the space of the main components of deformations (in Polish), Zeszyty Naukowe Politechniki Poznańskiej. Budowa Maszyn i Zarzadzanie Produkcja, 129-152 